\title{
Muertes prematuras y años de vida potenciales perdidos, ¿qué ha cambiado en Colombia durante la pandemia por COVID-19?
}

\author{
Premature deaths and years of potential life lost, what has \\ changed in Colombia during the COVID-19 pandemic?
}

\author{
Maylen-Liseth Rojas-Botero'; Julián-Alfredo Fernández-Niño ${ }^{1}$; Angélica-Nohelia Molina-Rivera ${ }^{1}$; Fernando Ruiz-Gómez ${ }^{1}$
}

Forma de citar: Rojas-Botero ML, Fernández-Niño JA, Molina-Rivera AN, Ruiz-Gómez F. Muertes prematuras y años de vida potenciales perdidos, ¿qué ha cambiado en Colombia durante la pandemia por COVID-19? Salud UIS. 52(4): 414-421. doi: https://doi.org/10.18273/revsal.v52n4-2020008 @c) (1)

\section{Resumen}

Introducción: la pandemia por COVID-19 es uno de los mayores desafíos globales de la época. Para la mayor comprensión de sus efectos poblacionales es necesario analizar medidas complentarias a la mortalidad. Objetivos: estimar los años de vida potenciales perdidos en Colombia debido a muertes prematuras por todas las causas de defunción y por COVID-19 en el periodo marzo-julio entre los años 2015 y 2020. Materiales y métodos: estudio ecológico, longitudinal y retrospectivo, basado en fuentes de información secundaria. Se calcularon los años de vida potencialmente perdidos según sexo y grupo de edad, utilizando la esperanza de vida y las tablas actuariales del DANE. Resultados: Entre marzo y julio de 2020 se perdieron 2356420 años por muertes prematuras, para una tasa de 46,8 años perdidos por cada mil habitantes de Colombia, un resultado superior en un 4,8\% respecto a la media de los últimos cinco años. Debido al COVID-19 se dejaron de vivir 237 725,5 años -con mayor pérdida entre los hombres-y un aporte porcentual del 10,5\% al total de años potenciales perdidos en Colombia. Conclusiones: Durante la pandemia se ha presentado un ligero incremento en los años de vida potenciales perdidos en Colombia. Si bien la pérdida de años atribuibles a las muertes confirmadas por COVID-19 no son los responsables directos de la mayor parte de la pérdida total, los cambios sociales y las condiciones de vida durante el confinamiento sí podrían haber incidido en las variaciones de la mortalidad y su distribución entre subgrupos poblacionales.

Palabras clave: Mortalidad prematura; Causa de muerte; Esperanza de vida; Tendencias; COVID-19; Colombia.

\begin{abstract}
Introduction: The COVID-19 pandemic is one of the greatest global challenges of the time. For a better understanding of its population effects, it is necessary to analyze complementary measures to mortality. Objetive: To estimate the potential years of life lost in Colombia due to premature deaths from all causes and from COVID-19 in the March-July period between 2015 and 2020. Materials and methods: We carried out an ecological, longitudinal and

1. Ministerio de Salud y Protección Social. Bogotá, Colombia.

Correspondencia: Julián Alfredo Fernández-Niño. Dirección: Carrera 13 No. 32-76, Teusaquillo, Bogotá D.C. Teléfono: +57(1) 3305000 ext 3122. Correo electrónico: jfernandezn@minsalud.gov.co


retrospective study, based on secondary sources. Years of life potentially lost were calculated according to sex and age group, using life expectancy and the DANE actuarial tables. Results: Between March and July 2020, 2356420 years were lost due to premature deaths, for a rate of 46.8 years lost per thousand inhabitants of Colombia, a result that is $4.8 \%$ higher than the average of the last five years. Due to COVID-19, 237725.5 years were lost -with the greatest loss among men-and a percentage contribution of $10.5 \%$ to the total potential years of life lost in Colombia. Conclusions: During the pandemic there has been a slight increase in the potential years of life lost in Colombia. Although the loss of years attributable to deaths confirmed by COVID-19 are not directly responsible for most of the total loss, social changes and living conditions during confinement could have had an impact on variations in mortality and its distribution among population subgroups.

Keywords: Mortality; Premature; Cause of death; Life expectancy; Trends; COVID-19; Colombia.

\section{Introducción}

La pandemia por COVID-19 constituye uno de los mayores desafíos a escala global de esta época. Desde el inicio del brote epidémico en Wuhan (China) hasta la dispersión del Sars-CoV-2 en alrededor de 189 países, la humanidad se ha visto enfrentada a un novedoso virus altamente contagioso y potencialmente letal, que ha causado la muerte de más de un millón de personas en el mundo en un lapso de diez meses ${ }^{1}$. Al tiempo, las diferentes estrategias de confinamiento instauradas en los territorios han develado problemáticas estructurales y provocado importantes cambios en las dinámicas sociales, con consecuencias a nivel político, y en las condiciones socioeconómicas y de salud de la población, lo que evidencia que la pandemia va más allá de una crisis sanitaria.

Para el caso específico de Colombia, en el país se adoptaron medidas de preparación desde antes de identificar el primer caso en el territorio ${ }^{2}$ y tras su confirmación, el 6 de marzo de $2020^{3}$, se dio inicio a un trabajo conjunto y articulado, con estrategias de contención, mitigación y supresión para disminuir la transmisión del virus y proteger a la población con mayor riesgo de cuadros graves. A la fecha, se han detectado 970 mil casos, más de 29 mil muertes ${ }^{4}$ y una magnitud todavía desconocida de discapacidad asociada al COVID-19.

Para entender mejor las consecuencias de la pandemia, se ha recomendado utilizar medidas de impacto más precisas que la tasa de mortalidad específica. Así, el Ministerio de Salud y Protección Social estimó el exceso de mortalidad en Colombia en relación con la magnitud de la mortalidad general en los últimos cinco años $^{5}$, encontrando un descenso inicial -posiblemente explicado por la disminución de las defunciones por causas externas-, y un incremento posterior coincidente con la reapertura económica y flexibilización de la movilidad.
En este sentido, el análisis de los años de vida potenciales perdidos (AVPP) ofrece una visión complementaria sobre la pérdida que sufre la sociedad debido a las muertes que ocurren prematuramente ${ }^{6}$. Con el propósito de aportar a la comprensión de los efectos del COVID-19 y asumiendo las implicaciones que supone valorar impactos en medio de una pandemia que todavía no termina, se presenta la estimación de los AVPP por muerte prematura debido a todas las causas de defunción y por COVID-19, para el periodo marzojulio entre los años 2015 y 2020 . Se espera que este sea un insumo que ayude al mejor entendimiento, ya no solo de las pérdidas vitales atribuibles al COVID-19, sino del impacto que ha tenido -y continúa teniendo- la pandemia en la población colombiana.

\section{Materiales y métodos}

Se llevó a cabo un estudio ecológico, longitudinal y retrospectivo, con fuentes de información secundaria. La unidad de análisis corresponde al país en el tiempo, en el periodo 2015-2020. Se utilizaron las bases de datos anonimizadas de estadísticas vitales, defunciones no fetales (preliminares para 2019 y 2020) y las tablas actuariales locales con la esperanza de vida para cada grupo de edad y sexo, publicadas por el DANE.

Los AVPP no solo tienen en cuenta el número de muertes ocurridas en un lugar y tiempo determinado, sino que también incorporan la edad a la que las personas fallecen, asignando un peso diferente a las muertes según el momento vital en el que acontecen. El indicador se calculó para cada sexo por separado, como se indica a continuación,

$$
A V P P=\sum\left(L_{i} \times d_{i}\right)
$$

donde $L$ corresponde a la esperanza de vida para cada grupo de edad $i, i$ es el punto medio de los intervalos para cada grupo de edad, y $d$ es el número de muertes observadas en cada grupo de edad $i$. 
Inicialmente, el indicador se calculó considerando todas las causas de defunción, y luego, se replicó el análisis tomando en cuenta únicamente las muertes confirmadas por COVID-19 (código CIE-10 U07.1) posterior al proceso de verificación y reclasificación por autopsia verbal, realizada por la Comisión Intersectorial de Gestión de las Estadísticas Vitales?

Se reportaron las frecuencias absolutas (AVPP) y relativas (tasas de AVPP por cada mil habitantes) para el periodo marzo-julio entre los años 2015 y 2020. Para el cálculo de las tasas se utilizaron las proyecciones y retroproyecciones poblacionales para cada año y sexo, publicadas por el DANE.
Se calcularon proporciones, tasas y razones de AVPP, además del promedio acompañado del intervalo de confianza de $95 \%$; los resultados se muestran a través de gráficos de barras y de tendencias. Para el análisis de la información se utilizó el sofware StataMP ${ }^{\circledR}$ v14.

\section{Resultados}

Entre marzo y julio de 2020 se reportaron en Colombia 109791 muertes por todas las causas y grupos de edad, la mayoría de las cuales acontecieron en hombres $(56,4 \%)$ y adultos mayores de 65 años $(64,7 \%)$. Estas muertes representan 2356420 años que se dejaron de vivir por muerte prematura, para una tasa de 46,8 AVPP por cada mil habitantes (Tabla 1).

Tabla 1. Años de vida potenciales perdidos por todas las causas según sexo y grupo de edad. Colombia, marzo-julio, $2015-2020$.

\begin{tabular}{|c|c|c|c|c|c|c|c|c|}
\hline \multirow{2}{*}{$\begin{array}{c}\text { Edad en } \\
\text { años }\end{array}$} & \multicolumn{2}{|c|}{ Hombres } & \multicolumn{2}{|c|}{ Mujeres } & \multicolumn{2}{|c|}{ Total 2020} & \multirow{2}{*}{$\begin{array}{c}\text { 2015-2019 } \\
\text { Tasa } \\
\text { media AVPP }\end{array}$} & \multirow[b]{2}{*}{ Diferencia porcentual } \\
\hline & AVPP & $\begin{array}{c}\text { Tasa } \\
\text { AVPP }\end{array}$ & AVPP & $\begin{array}{c}\text { Tasa } \\
\text { AVPP }\end{array}$ & AVPP & $\begin{array}{c}\text { Tasa } \\
\text { AVPP }\end{array}$ & & \\
\hline $0-4$ & 128060,8 & 63,8 & 99807,7 & 52,0 & 227868 & 58,0 & 72,5 & $-19,9 \%$ \\
\hline $5-9$ & 11096,3 & 5,5 & 8469,3 & 4,4 & 19565,6 & 5,0 & 6,6 & $-25,2 \%$ \\
\hline $10-14$ & 16795,0 & 8,3 & 10922,7 & 5,6 & 27717,6 & 7,0 & 7,6 & $-7,7 \%$ \\
\hline $15-19$ & 53378,6 & 25,4 & 19218,5 & 9,5 & 72597,1 & 17,6 & 23,0 & $-23,8 \%$ \\
\hline $20-24$ & 89775,89 & 41,2 & 27742,5 & 12,9 & 117518 & 27,2 & 31,4 & $-13,6 \%$ \\
\hline $25-29$ & 85774,0 & 40,8 & 30455,5 & 14,3 & 116230 & 27,5 & 30,2 & $-8,9 \%$ \\
\hline $30-34$ & 72935,5 & 38,3 & 31310,0 & 15,9 & 104246 & 27,0 & 28,9 & $-6,8 \%$ \\
\hline $35-39$ & 68215,8 & 38,9 & 38303,5 & 20,7 & 106519 & 29,6 & 28,6 & $3,3 \%$ \\
\hline $40-44$ & 64826,7 & 42,0 & 41960,4 & 25,0 & 106787 & 33,2 & 29,9 & $10,9 \%$ \\
\hline $45-49$ & 66726,0 & 48,6 & 48332,8 & 31,5 & 115059 & 39,6 & 34,4 & $15,0 \%$ \\
\hline $50-54$ & 80181,0 & 60,6 & 60808,3 & 40,2 & 140989 & 49,7 & 42,7 & $16,5 \%$ \\
\hline $55-59$ & 95734,8 & 79,6 & 73139,2 & 52,3 & 168874 & 65,0 & 55,7 & $16,5 \%$ \\
\hline $60-64$ & 103797,4 & 105,4 & 81921,8 & 70,5 & 185719 & 86,5 & 74,2 & $16,6 \%$ \\
\hline $65-69$ & 102886,2 & 137,0 & 80340,3 & 89,5 & 183226 & 111,1 & 93,8 & $18,4 \%$ \\
\hline $70-74$ & 94542,5 & 175,0 & 74171,8 & 112,6 & 168714 & 140,7 & 122,0 & $15,4 \%$ \\
\hline $75-79$ & 76157,1 & 213,2 & 67190,8 & 150,1 & 143348 & 178,1 & 171,8 & $3,7 \%$ \\
\hline 80 y más & 158066,3 & 364,9 & 193375,4 & 335,5 & 351442 & 348,1 & 334,5 & $4,1 \%$ \\
\hline Total & 1368949,9 & 55,7 & 987470,5 & 38,3 & 2356420 & 46,8 & 44,6 & $4,8 \%$ \\
\hline
\end{tabular}

*Datos preliminares para 2019 y 2020 . Tasas por mil habitantes.

Al comparar con los datos históricos, la tasa de AVPP global fue superior en un 4,8\% en relación con la media de AVPP para los últimos cinco años (AVPP por cada mil habitantes), con variaciones según los grupos de edad. Así, los mayores incrementos se observaron entre los adultos a partir de los 40 años. La variación porcentual para los hombres fue ligeramente superior en relación con las mujeres (aumento del 5,2\% y del $4,3 \%$ en los AVPP respectivamente); además, se observaron diferencias de acuerdo con la edad en ambas subpoblaciones (Figura 1). 


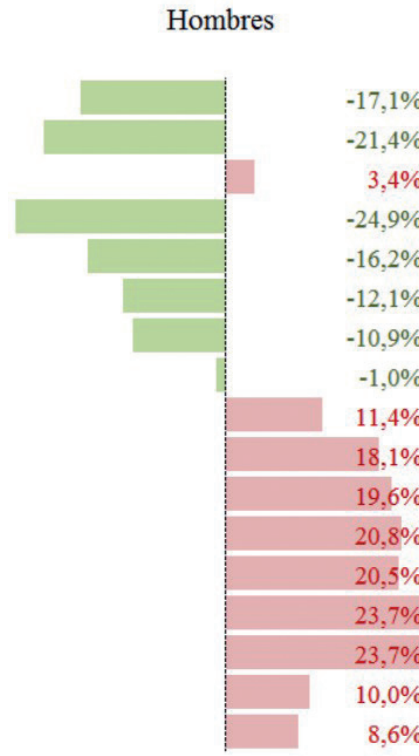

$0-4$
$5-9$
$10-14$
$15-19$
$20-24$
$25-29$
$30-34$
$35-39$
$40-44$
$45-49$
$50-54$
$55-59$
$60-64$
$65-69$
$70-74$
$75-79$
80 y más

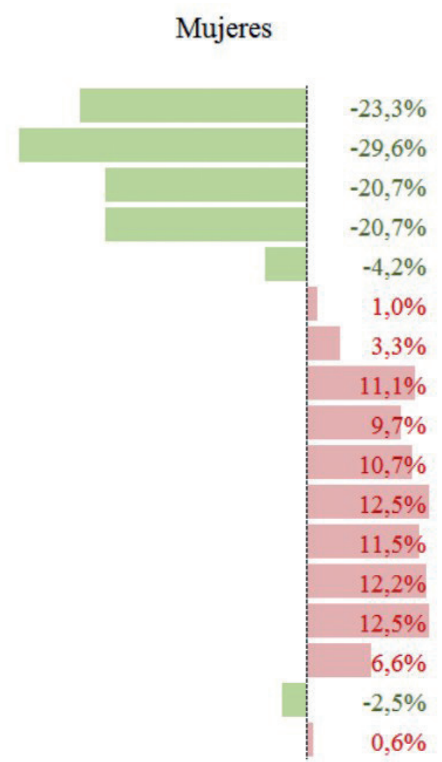

Figura 1. Variación porcentual de los AVPP por todas las causas en 2020 respecto a la media histórica para el periodo marzojulio, según grupos de edad y sexo.

*Datos preliminares para 2019 y 2020.

El análisis de tendencias muestra una magnitud estable en los AVPP por cada mil habitantes, con excepción del año 2017, cuando se presentó un descenso considerable tanto para los hombres como para las mujeres y para todos los grupos etarios. Como se observa en la Figura 2a, la mayor pérdida de años potenciales de vida entre los hombres se presentó en toda la serie de estudio.

Entre tanto, y según grupos de edad, fueron los adultos mayores de 80 años quienes más aportaron a los AVPP, seguidos muy cerca por los niños menores de un año. Nótese que la tendencia observada en la Figura 2b sugiere para el año 2020 un leve incremento en los

a. Tendencia de los AVPP según sexo

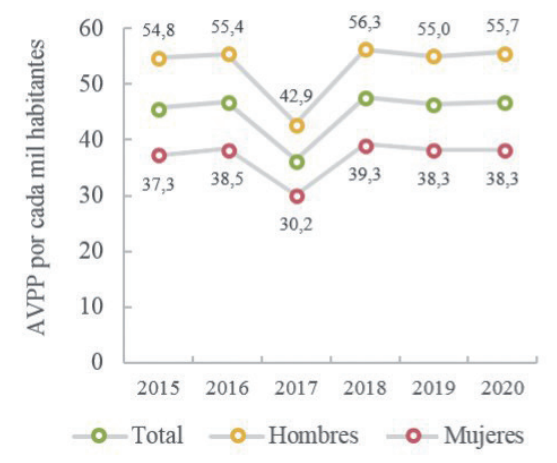

AVPP de los adultos entre 45 y 64 años, lo mismo que para los adultos entre 65 y 79 años, y para los mayores de 80; caso contrario a lo ocurrido entre los niños menores de un año -con una disminución del 19,5\% con respecto al año inmediatamente anterior-, y entre los niños, adolescentes y adultos menores de 44 años.

$\mathrm{Al}$ considerar exclusivamente las muertes confirmadas por COVID-19, entre marzo y julio de 2020 se perdieron en Colombia 237 725,5 años potenciales de vida en la población (Tabla 2), es decir, 4,7 AVPP por cada mil habitantes -estos aportaron el 10,5\% de los AVPP totales observados en el periodo- .

b. Tendencia de los AVPP según grupos

de edad

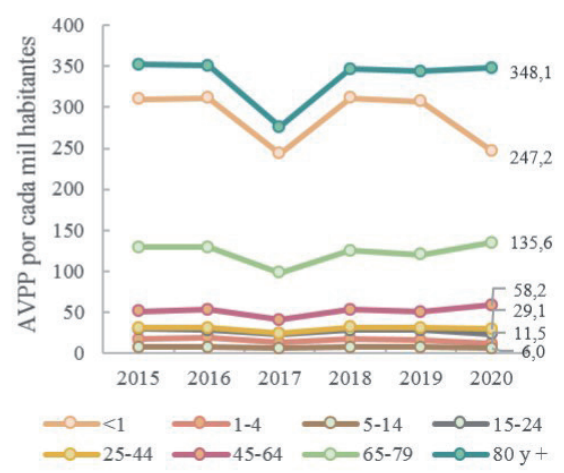

Figura 2. Tendencias de los AVPP por todas las causas de muerte según sexo y grupos de edad. Colombia, marzo-julio, 2015-2020* *Datos preliminares para 2019 y 2020. 
Tabla 2. Años de vida potenciales perdidos por COVID-19 por cada mil habitantes según sexo y grupo de edad. Colombia, marzo-julio de 2020.

\begin{tabular}{ccccccccccc}
\hline \multirow{2}{*}{$\begin{array}{c}\text { Edad en } \\
\text { años }\end{array}$} & \multicolumn{3}{c}{ Hombres } & \multicolumn{1}{c}{ Mujeres } & \multicolumn{3}{c}{ Total } \\
\cline { 2 - 10 } & $\mathbf{n}$ & $\mathbf{E V}$ & AVPP & Tasa AVPP & $\mathbf{n}$ & $\mathbf{E V}$ & AVPP & Tasa AVPP & AVPP & Tasa AVPP \\
\hline $0-4$ & 25 & 73,8 & 1844,6 & 0,9 & 15 & 80,3 & 1204,5 & 0,6 & 3049,1 & 0,8 \\
$5-9$ & 6 & 70,2 & 421,4 & 0,2 & 4 & 76,3 & 305,2 & 0,2 & 726,6 & 0,2 \\
$10-14$ & 5 & 65,4 & 326,8 & 0,2 & 1 & 71,39 & 71,4 & 0,0 & 398,1 & 0,1 \\
$15-19$ & 12 & 60,5 & 726,2 & 0,3 & 8 & 66,5 & 532,0 & 0,3 & 1258,2 & 0,3 \\
$20-24$ & 36 & 56,0 & 2014,9 & 0,9 & 19 & 61,65 & 1171,4 & 0,5 & 3186,3 & 0,7 \\
$25-29$ & 50 & 51,6 & 2582,0 & 1,2 & 43 & 56,82 & 2443,3 & 1,2 & 5025,3 & 1,2 \\
$30-34$ & 86 & 47,3 & 4070,4 & 2,1 & 43 & 52,01 & 2236,4 & 1,1 & 6306,8 & 1,6 \\
$35-39$ & 169 & 42,9 & 7255,2 & 4,1 & 75 & 47,23 & 3542,3 & 1,9 & 10797,4 & 3,0 \\
$40-44$ & 251 & 38,5 & 9651,0 & 6,3 & 107 & 42,47 & 4544,3 & 2,7 & 14195,2 & 4,4 \\
$45-49$ & 376 & 33,9 & 12761,4 & 9,3 & 123 & 37,76 & 4644,5 & 3,0 & 17405,9 & 6,0 \\
$50-54$ & 481 & 29,5 & 14189,5 & 10,7 & 214 & 33,12 & 7087,7 & 4,7 & 21277,2 & 7,5 \\
$55-59$ & 697 & 25,2 & 17564,4 & 14,6 & 311 & 28,57 & 8885,3 & 6,4 & 26449,7 & 10,2 \\
$60-64$ & 849 & 21,1 & 17947,9 & 18,2 & 438 & 24,18 & 10590,8 & 9,1 & 28538,7 & 13,3 \\
$65-69$ & 1017 & 17,4 & 17695,8 & 23,6 & 527 & 20,02 & 10550,5 & 11,8 & 28246,3 & 17,1 \\
$70-74$ & 1059 & 14,1 & 14879,0 & 27,5 & 566 & 16,17 & 9152,2 & 13,9 & 24031,2 & 20,0 \\
$75-79$ & 923 & 11,2 & 10309,9 & 28,9 & 557 & 12,74 & 7096,2 & 15,9 & 17406,1 & 21,6 \\
80 y más & 1816 & 8,8 & 15944,5 & 36,8 & 1373 & 9,82 & 13482,9 & 23,4 & 29427,3 & 29,2 \\
Total & 7858 & & 150184,8 & 6,1 & 4424 & & 87540,7 & 3,4 & 237725,5 & 4,7 \\
\hline
\end{tabular}

Datos preliminares. $\mathrm{n}=$ número de muertes. $\mathrm{EV}=$ Esperanza de vida. $\mathrm{AVPP}=\mathrm{Años}$ de vida potenciales perdidos. Tasas por mil habitantes.

Invariablemente, la pérdida fue superior entre los hombres en comparación con las mujeres en todos los grupos etarios, con una brecha que aumentó conforme aumentaba la edad. Esta diferencia fue del 79,8\%, de manera que por cada diez años de vida potenciales perdidos por COVID-19 entre las mujeres, se perdieron aproximadamente 18 años entre los hombres por la misma enfermedad.

Finalmente, como puede verse en la Figura 3, la pérdida de años potenciales de vida se incrementa considerablemente a partir de los 45 años de edad, con un valor máximo entre los adultos de 80 y más años; con mayor participación en los AVPP totales conforme va aumentando la edad.

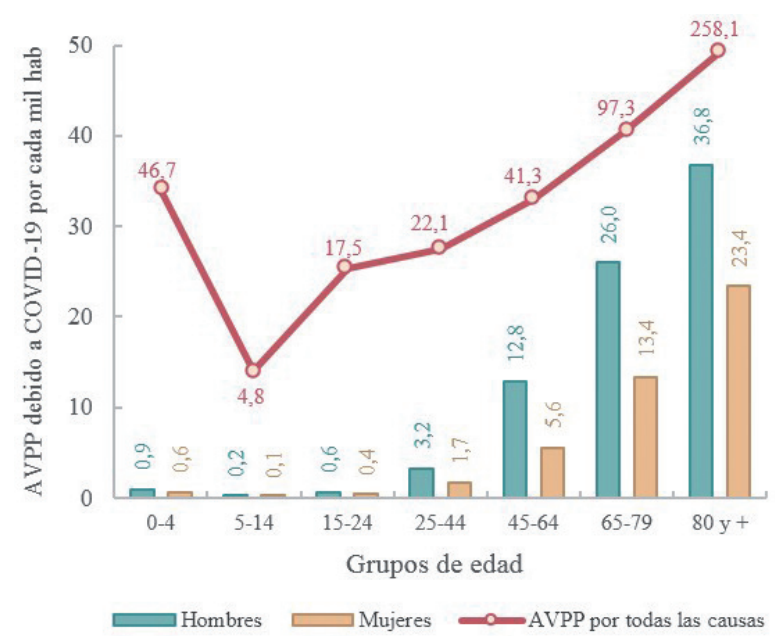

Figura 3. AVPP por COVID-19 confirmado según sexo y grupos de edad, y comparación con los AVPP por todas las causas para cada grupo de edad. Colombia, marzo-julio de 2020.

*Datos preliminares para 2020. 


\section{Discusión}

El surgimiento del COVID-19 como enfermedad novedosa, potencialmente letal y altamente contagiosa entre los seres humanos ha provocado una crisis global y multisectorial. Desde su introducción a Colombia, se han confirmado más de 29 mil muertes por su causa, con una mayor carga entre los adultos mayores $(75,9 \%)$ y entre los hombres $(64,2 \%)^{4}$. No obstante, para comprender mejor el impacto global de la pandemia en términos de pérdidas vitales, no solo se deben considerar las muertes confirmadas, sino también las sospechosas y las muertes ocurridas por otras causas ${ }^{8,9}$.

En esa vía, este estudio ofrece el cálculo de los AVPP por todas las causas de defunción antes y durante la pandemia en Colombia. Se encontró que entre el $1^{\circ}$ de marzo y el 31 de julio de 2020 , se perdieron 2356420 años potenciales de vida, lo cual representa un incremento del 4,8\% con relación al promedio de los últimos cinco años para el mismo periodo, con un comportamiento heterogéneo según grupos de edad y sexo. Si bien el incremento se observó tanto para los hombres como para las mujeres, este fue ligeramente mayor en la población masculina. Entre tanto, cuando se consideró la edad, el aumento fue destacable solo a partir de los 40 años en el caso de los hombres, y de los 35 entre las mujeres.

En el caso específico del virus, el COVID-19 ha sido responsable de la pérdida directa de 237725,5 años potenciales de vida, es decir que las muertes por COVID-19 aportaron el 10,5\% de todos los años de vida perdidos en el país durante marzo y julio de 2020; el 89,5\% restante correspondió al aporte de otras causas de muerte, cuya magnitud y distribución también pudieron verse modificados a partir de los cambios en la dinámica social originados por la pandemia, por ejemplo, algunas causas externas de mortalidad.

Es posible que la cantidad de muertes debido a incidentes viales haya disminuido considerablemente debido a la reducción de la movilidad vehicular, máxime si se considera que la mayoría de las muertes viales ocurren en personas jóvenes ${ }^{10,11}$. En contraste, es posible que haya aumentado el número de feminicidios y agresiones contra niños y niñas, en la medida que este tipo de violaciones a los derechos humanos se han incrementado con anterioridad durante epidemias como la del Zika y el Ébola ${ }^{12}$ y ya han sido advertidos en varios contextos durante esta pandemia ${ }^{13,14}$.
Un mayor aporte a los AVPP puede deberse a un elevado número de muertes, a la defunción de personas jóvenes o a la combinación de ambas. En este sentido, la magnitud de las muertes confirmadas por COVID-19 en Colombia no se refleja en un incremento proporcional en los AVPP, esto debido a la edad de quienes han fallecido por esta causa $-\mathrm{y}$ a su menor esperanza de vida $-\mathrm{y}$ a la reducción de eventos mortales en la población joven, posiblemente relacionada con la combinación de estrategias de cuarentena, aislamiento, distanciamiento social y confinamiento para la reducción de la transmisión del virus, cuyos efectos indirectos todavía están por evaluarse.

En este estudio la tasa de AVPP fue superior entre los hombres en comparación con las mujeres, esta diferencia fue del $45,3 \%$ para todas las causas de muerte, y del 80,0\% para COVID-19. De acuerdo con la Organización Mundial de la Salud (OMS), las pandemias presentan efectos diferenciales entre hombres y mujeres, originados por sus características biológicas, grado de exposición e interacción con otros determinantes sociales de la salud ${ }^{15}$. Por otro lado, la evidencia disponible sobre epidemias antecedentes muestran diferencias de sexo y género en relación con la sensibilidad al virus y vulnerabilidad a la infección, que deberían considerarse durante la elaboración, ejecución y evaluación de programas sobre enfermedades infecciosas emergentes ${ }^{16}$.

Esta mayor pérdida de años potenciales de vida entre los hombres fue también reportada por Oh et al. para Alemania, Brasil, Chile, Ecuador, España, Estados Unidos, Francia, Irán, Italia, Perú, Portugal, Reino Unido, Rumania, Rusia, Suiza y Turquía en el estudio de los AVPP atribuibles al COVID-19 en países de alta incidencia de la enfermedad ${ }^{17}$.

Este trabajo presenta algunas limitaciones, entre ellas, las concernientes a la utilización de fuentes secundarias de información. Pueden existir errores de clasificación (codificación) de las causas de muerte, especialmente entre las muertes con diagnóstico clínico-epidemiológico de COVID-19, en las que no se logra identificar el virus y son consideradas como casos probables o sospechosos. Sin embargo, debe advertirse que en Colombia se siguen las orientaciones internacionales para la certificación y clasificación del COVID-19 como causa de muerte ${ }^{18}$ y se lleva a cabo un proceso riguroso de verificación, reclasificación y autopsia verbal, realizada por la Comisión Intersectorial 
de Gestión de las Estadísticas Vitales como parte de la vigilancia epidemiológica y demográfica de la mortalidad por COVID-197.

Por otro lado, se utilizó la esperanza de vida para cada grupo de edad proveniente de las tablas actuariales de la población Colombiana. Si bien este criterio metodológico permitió una aproximación más precisa a la pérdida de años potenciales de vida dada la expectativa de vida en el país, debe considerarse para realizar con mesura las comparaciones con poblaciones internacionales.

Se espera que los resultados ofrecidos en este artículo aporten a un mejor entendimiento de los efectos del COVID-19 en el país, ya no solo desde la mortalidad atribuible directamente al virus, sino a las pérdidas vitales y años que dejaron de vivirse debido a las modificaciones en las condiciones de vida ocasionadas por la pandemia.

\section{Agradecimientos}

Al Ministerio de Salud y Protección Social por la financiación.

\section{Consideraciones éticas}

Al tratarse de un estudio con fuentes de información secundarias, no se requirió el aval de un comité de ética de la investigación. En todo momento se siguieron los principios éticos establecidos en la Resolución 8430 de $1993^{19}$, entre tanto, al utilizar bases de datos agregadas se garantizó el anonimato de la población.

\section{Conflicto de interés}

No se declaran conflictos de intereses.

\section{Referencias}

1. Johns Hopkins University \& Medicine. Coronavirus resource center. Johns Hopkins Coronavirus Resource Center. 2020. https://coronavirus.jhu.edu/ map.html

2. MinSalud. Circular externa 05 de 2020. Directrices para la detección temprana, el control y la atención ante la posible introducción del nuevo Coronavirus (2019-nCoV) y la implementación de los planes de preparación y respuesta ante este riesgo. Ministerio de Salud y la Protección Social; 2020.
3. MinSalud. Colombia confirma su primer caso de COVID-19. Boletín de prensa no 050 de 2020. 2020. https://www.minsalud.gov.co/Paginas/Colombiaconfirma-su-primer-caso-de-COVID-19.aspx

4. INS. Covid-19 en Colombia. Reporte 18-10-2020. COVID-19 en Colombia. 2020. https://www.ins. gov.co/Noticias/paginas/coronavirus.aspx

5. MinSalud. Exceso de mortalidad en Colombia, 2020. MinSalud; 2020. https://www.minsalud. gov.co/sites/rid/Lists/BibliotecaDigital/RIDE/ $\mathrm{VS} / \mathrm{ED} / \mathrm{VSP} /$ estimacion-exceso-mortalidadColombia-2020.pdf

6. OPS. Técnicas para la medición del impacto de la mortalidad: Años Potenciales de Vida Perdidos. Boletín Epidemiológico. 2003. https://www. uv.es/ 1sn/BIOTICS/Demo/Apunts/doc/APVP/be v24n2-APVP.htm

7. Ministerio de Salud y Protección Social. Guía para el reporte y verificación de las defunciones en SegCOVID-19. MinSalud; 2020. https://www. minsalud.gov.co/sites/rid/Lists/BibliotecaDigital/ $\mathrm{RIDE} / \mathrm{VS} / \mathrm{ED} / \mathrm{VSP} / \mathrm{pspg}$ 04guia-reporte-verifiaciondefunciones-segcovid.pdf

8. Beaney T, Clarke JM, Jain V, Golestaneh AK, Lyons G, Salman D, et al. Excess mortality: the gold standard in measuring the impact of COVID-19 worldwide? J R Soc Med. 2020; 113(9): 329-34. doi: https://doi.org/10.1177/0141076820956802

9. Woolf SH, Chapman DA, Sabo RT, Weinberger DM, Hill L. Excess Deaths From COVID-19 and Other Causes, March-April 2020. JAMA. 2020; 324(5): 510-3. doi: 10.1001/jama.2020.11787

10. International Transport Forum. Road safety annual report 2019. International Transport Forum; 2019. https://www.itf-oecd.org/sites/default/files/docs/ irtad-road-safety-annual-report-2019.pdf

11. Domingo Alarcón J, Gich Saladich I, Vallejo Cuellar L, Ríos Gallardo AM, Montalvo Arce C, Bonfill Cosp X, et al. Mortalidad por accidentes de tráfico en Colombia. Estudio comparativo con otros países. Rev Esp Salud Publica. 2018; 92: 01807040.

12. Roesch E, Amin A, Gupta J, García-Moreno C. Violence against women during covid-19 pandemic restrictions. BMJ. 2020; 369. doi: https://doi. org/10.1136/bmj.m1712

13. Telles LE de B, Valença AM, Barros AJS, da Silva AG, Telles LE de B, Valença AM, et al. Domestic violence in the COVID-19 pandemic: a forensic psychiatric perspective. Braz J Psychiatry. 2020; ahead of print. doi: https://doi.org/10.1590/15164446-2020-1060 
14. Lund RG, Manica S, Manica G. Collateral issues in times of covid-19: child abuse, domestic violence and femicide. RBOL. 2020; 7(2): 54-69. doi: http:// orcid.org/0000-0002-8352-2888

15. OMS. El género y la COVID-19. Organización Mundial de la Salud; 2020. https:/apps.who. int/iris/bitstream/handle/10665/332242/WHO2019-nCoV-Advocacy_brief-Gender-2020.1-spa. pdf? sequence $=1 \&$ isAllowed $=y$

16. World Health Organization, Regional Office for the Western Pacific. Taking sex and gender into account in emerging infectious disease programmes: an analytical framework. 1a ed. Washington D.C.: WHO; 2011. http://iris.wpro.who.int/ handle/10665.1/7977

17. Oh I-H, Ock M, Jang SY, Go D-S, Kim Y-E, Jung Y-S, et al. Years of Life Lost Attributable to COVID-19 in High-incidence Countries. J Korean Med Sci. 2020; 35(32). doi: 10.3346/jkms.2020.35.e300

18. OPS. Orientación internacional para la certificación y clasificación (codificación) del COVID-19 como causa de muerte. Basada en la CIE. Organizacion Panamericana de la Salud; 2020. https://www. who.int/classifications/icd/Guidelines_Cause_of_ Death_COVID-19-20200423_ES.pdf?ua =1

19. República de Colombia, Ministerio de Salud. Resolución No 008430 de 1993. Por la cual se establecen las normas científicas, técnicas y administrativas para la investigación en salud. 008430 de 1993.1993 p. 24. 